\title{
PREVALENCIA DE MARCADORES INFECCIOSOS EN DONANTES DE SANGRE
}

\author{
PREVALENCE OF VIRAL MARKERS IN BLOOD DONORS
}

\author{
Tucto-López Olga ${ }^{1, a, b}$ \\ 1. Universidad Nacional San Luis Gonzaga de Ica, Perú \\ a. Médico Residente Patología Clínica \\ b. Médico Cirujano
}

doi: https://doi.org/10.35563/rmp.v8i2.6

\section{Correspondencia:}

Olga Tucto López

Dirección: Urb. San Joaquín ॥

Etapa KI-I

Número de celular: +51 985534642

Correo Electrónico:

tuctoolga@gmail.com

Contribuciones De Autoría:

OTL Contribuciones en la concepción y diseño del manuscrito, recolección, análisis e interpretación de los datos, redacción y revisión crítica del contenido del manuscrito y aprobación final del artículo

Conflicto De Intereses: No declarados.

Financiamiento: Autofinanciado.

\section{Como Citar}

Tucto-López Olga. Prevalencia de marcadores infecciosos en donantes de sangre. Rev méd panacea. 2019; (2):78-81. doi: https://doi.org/10.35563/rmp.v8 i2.275

Recibido: 10 - 03 - 2019

Aceptado: 09 - 05 - 2019

Publicado: 12 - 05 - 2019

\section{RESUMEN}

Objetivo: El objetivo de este artículo es realizar una revisión actualizada sobre prevalencia de marcadores virales en donantes del Banco de sangre. Materiales y métodos: La búsqueda bibliografía se ha realizado en Pubmed, Medline y Scielo. Como criterios de selección se tomó en cuenta información publicada en dichas fuentes dada la confiabilidad de las mismas, con una antigüedad no mayor de 5 años, tanto en idioma español como inglés. Resultados: Se encontró un total de seis artículos: en México, se encontró un descenso de 5,375 (prevalencia=0.47) a 2,675 (prevalencia $=0.15$ ) casos seropositivos para VHB. El marcador serológico del VHC presentó un aumento de 8,170 (prevalencia=0.69) a 10,217 (prevalencia=0.57). En Colombia el marcador infeccioso más frecuentemente encontrado en los donantes fue Sífilis (1,73\%), seguido por Hepatitis B (1,53\%), Hepatitis C $(0,46 \%)$ HTLV I-II $(0,21 \%)$, la enfermedad de Chagas $(0,27 \%)$ y VIH $(0,17 \%)$. En Cuba se encontró una prevalencia de AgsHB 1,0\%, VHC 2,3\%, VIH 3,4 \% y sifilis 2,3\%. En Perú el 0,6\% $(n=156)$ fue reactivo para HBsAg; $5,2 \%(n=1465)$ para anti-HBcAb 0,8\% $(n=232)$ para Anti-HVC y $1.21 \%$ para HTLV I-II. Conclusiones: Las cifras de prevalencia reportadas en los estudios son coherentes con los reportes de la OPS y coinciden entre ellos.

La prevalencia constante de estos marcadores se debe a la poca educación sanitaria al respecto de las enfermedades transmisibles en cuanto a su transmisibilidad, diagnóstico y tratamiento oportuno.

Palabras clave: prevalencia; donantes de sangre; marcadores serología (fuente: DeCS BIREME).

\section{ABSTRACT}

Objective: The objective of this article is to carry out an updated review on the prevalence of viral markers in Blood Bank donors. Materials and methods: The bibliography search was carried out in Pubmed, Medline and Scielo. As criteria for selection, information published in said sources was taken into account given the reliability of the same, with an age of no more than 5 years, in both Spanish and English. Results: Six articles were found: in Mexico, a decrease of 5.375 (prevalence = 0.47 ) to 2.675 (prevalence $=0.15$ ) was found for seropositive cases for HBV. The serological marker of HCV showed an increase of 8,170 (prevalence $=0.69$ ) to 10,217 (prevalence $=0.57$ ). In Colombia, the infectious marker most frequently found in the donors was Syphilis (1.73\%), followed by Hepatitis B (1.53\%), Hepatitis C (0.46\%), HTLV I-II $(0.21 \%)$, Chagas disease $(0.27 \%)$ and HIV $(0.17 \%)$. In Cuba, a prevalence of HBsAg 1.0\%, HCV 2.3\%, HIV 3.4\% and syphilis $2.3 \%$ was found. In Peru, $0.6 \%(n=156)$ was reactive for HBsAg; $5.2 \%(n=1465)$ for anti-HBcAb 0.8\% $(n=232)$ for Anti-HVC and $1.21 \%$ for HTLV I-II. Conclusions: The prevalence figures reported in the studies are consistent with the PAHO reports and coincide among them.

The constant prevalence of these markers is due to the lack of health education regarding communicable diseases in terms of their transmissibility, diagnosis and timely treatment.

Keywords: prevalence; blood donors; serology (source: MeSH NLM). 


\section{INTRODUCCIÓN}

La transfusión sanguínea es una estrategia utilizada frecuentemente con propósitos terapéuticos, y en ocasiones con fines preventivos, en diferentes escenarios hospitalarios. Anteriormente, las complicaciones más graves eran la transmisión de enfermedades infecciosas y las reacciones hemolíticas por incompatibilidad $A B O$, que con el avance de técnicas de laboratorio y el diseño de protocolos para donación y administración de las transfusiones han disminuido significativamente su frecuencia y han permitido incrementar ostensiblemente la seguridad del paciente (1).

Actualmente al realizar transfusiones sanguíneas no es posible asegurar ningún riesgo. Para el año 2009 la organización mundial de la salud reportó una prevalencia de 33 millones de personas infectadas con Virus de la Inmunodeficiencia Humana $(\mathrm{VIH})$ a nivel mundial, y se estima que más de 350 millones de personas presentan Hepatitis B (VHB), Hepatitis C (VHC) o ambas (2).

En países con un Índice de Desarrollo Humano medio y alto se presentan bajos índices de transmisión de $\mathrm{VIH}$, virus de la hepatitis B y C (1 por cada 1500 000, 1 por cada 282 000, y 1 por cada 2000000 de habitantes, respectivamente). La relación del Índice de Desarrollo Humano con la prevalencia de enfermedades transmitidas por transfusión sanguínea para países con un Índice de Desarrollo Humano alto, medio y bajo es de $0,001 \%, 0,06 \%$ y $0,5 \%$, respectivamente (3).

En concordancia con lo anterior, se conoce que el riesgo de contaminación con la transfusión de una unidad de sangre es de 1 en 132000 para el VIH, 1 en 43000 para la hepatitis B y 1 en 19000 para la hepatitis C. Para el VIH y el Virus de la Hepatitis B (VHB), por lo menos $90 \%$ del riesgo es atribuible al periodo de ventana; mientras que para el Virus de la Hepatitis C (VHC) es de 73\% a 88\% (4). La Organización Mundial de la Salud establece que a toda unidad de sangre y hemocomponentes se le debe realizar pruebas de tamizaje, para la detección de marcadores serológicos de infecciones transmisibles por transfusiones sanguíneas. Actualmente en el Perú se realizan pruebas de tamizaje para la detección de anticuerpos contra el virus de la hepatitis $C$, del antígeno de superficie de la hepatitis $B$, de los virus linfotrópicos de células $T$ humanas (HTLV-1 Y HTLV-2), antígeno y anticuerpo contra el virus del VIH (5).

Ante la mayor demanda de componentes sanguíneos por razones terapéuticas médicas y conociendo que existen muchas infecciones que transmiten por transfusión sanguínea. El objetivo de este artículo es realizar una revisión actualizada sobre prevalencia de marcadores virales en donantes del Banco de sangre.

\section{MATERIALES Y MÉTODOS}

La búsqueda bibliografía se ha realizado en Pubmed, Medline y Scielo. Como criterios de selección se tomó en cuenta información publicada en dichas fuentes dada la confiabilidad de las mismas, con una antigüedad no mayor de 5 años, tanto en idioma español como inglés.

\section{RESULTADOS}

Se encontró un total de seis artículos, siendo tres de ellos realizados en población americana, dentro de ellos dos estudios peruanos.

J.Rojo-medina y JMBello-López (Mexico, 2017) realizaron un estudio de prevalencia de los virus de hepatitis $C$ (VHC) y $\mathrm{B}(\mathrm{VHB})$ en donantes de sangre mexicanos, encontrando que de un total de 19,096 294 y 18,617 288 informes mensuales con resultados de pruebas serológicas del VHB y VHC fueron obtenidos respectivamente durante 13 años (2000-2012). Un descenso de 5,375 (prevalencia=0.47) a 2,675 (prevalencia $=0.15$ ) casos seropositivos al VHB fue observado. El marcador serológico del VHC presentó un aumento de 8,170 (prevalencia=0.69) a 10,217 (prevalencia $=0.57$ ) casos seropositivos. Concluyen que el aumento de la prevalencia del VHC indica que sigue siendo un potencial patógeno transmisible por transfusión y necesita tratamiento oportuno y eficaz (7). Leidy Camargo De la Hoz y col. (Colombia, 2018) realizaron un estudio para determinar el perfil de donantes de sangre y seroprevalencia de marcadores infecciosos, encontrando que $78,86 \%$ de los donantes de sangre fueron vo $\neg$ luntarios por primera vez, las participación de la mujer como donante fue significativamente más baja (32,69\%) en com-paración con los hombres (67,31\%), la fuente más impor $\neg$ tante de donantes fue la captación en campañas de dona $\neg$ ción (74,19\%). El marcador infeccioso más frecuentemente encontrado en los donantes fue Sífilis $(1,73 \%)$, seguido por Hepatitis B (1,53\%), Hepatitis C $(0,46 \%)$ HTLV I-II $(0,21 \%)$, la enfermedad de Chagas $(0,27 \%)$ y VIH $(0,17 \%)$ (8).

Jaiberth Antonio Cardona y col. (Colombia, 2018) realizaron un estudio de prevalencia de virus de las Hepatitis B y C y factores asociados en un banco de sangre con base en una población de 25.842 donantes, se halló una alta proporción de donaciones en adultos jóvenes (57\%) y mujeres (53\%), predominó el tipo altruista (75\%), no repetitiva o de primera vez (76\%). La prevalencia del VHB fue $1,5 \%$ y del VHC 0,40\% (9).

María Antonia Ramos Ríos y col. (Cuba, 2014) realizaron un estudio de incidencia de marcadores serológicos en donante de sangre en base a una población de 989 , se alcanzó una incidencia de AgsHB 1,0\%, VHC 2,3\%, VIH $3,4 \%$ y sifilis 2,3\% (10).

Jeél Moya y Edward Julcamanyan (Perú, 2014) realizaron un estudio de seroprevalencia de marcadores infecciosos causantes de pérdidas de hemodonaciones en el Servicio de Banco de Sangre, los hallazgos fueron Los hallazgos fueron $4.63 \%$ para $\mathrm{HBcAb}, 1.78 \%$ para sífilis, $1.21 \%$ para HTLV I-II, y $5.31 \%$ para otros marcadores serológicos de un total de 11399 donaciones completas. Concluyendo en que la prevalencia hallada demostró la mala calidad de donantes de sangre y el gran impacto económico por 
hemoderivados desechados muestran las limitaciones en la cadena de donación (11).

Juan Morales y Col. (Perú, 2017) realizaron un estudio con el objetivo de determinar la frecuencia de marcadores de infección para hepatitis $B$, hepatitis $C$ y conocerlos factores asociados en los donantes de sangre. El estudio se realizó con datos del registro de donantes de un hospital público de Lima. De 28263 sujetos analizados entre 2012 y 2015 , el $0,6 \%(n=156)$ fue reactivo para $\mathrm{HBsAg}$; $2 \%(n=1465)$ para anti-HBc, y 0,8\% ( $n=232)$ para Anti-HVC (12).

\section{DISCUSIÓN}

La gran mayoría de los agentes transmisibles por transfusión, en los que se incluyen los virus de las hepatitis B y C y los retrovirus humanos, se pueden transmitir por vía sexual y se mantienen por largos períodos, como infecciones asintomáticas o estados de portador. Además, en un donante cuya sangre se extrae durante el período de incubación, puede ser infectante. Al mantenerse ocultos los hábitos de comportamiento sexual, implica un riesgo adicional (10).

Entre los agentes infecciosos estudiados en los artículos revisados están el virus de la inmunodeficiencia humana $(\mathrm{VIH})$, virus de la hepatitis $B(\mathrm{VHB})$, virus de la hepatitis $C$ (VHC), virus linfotropico humano (HTLV) y sífilis.

La OPS reportó prevalencia de 0,19\%, 4,31\%, 0,47\%, $1,12 \%$ y $0,61 \%$ para $\mathrm{VIH}, \mathrm{VHB}, \mathrm{VHC}$, sífilis y Chagas, respectivamente, en unidades de sangre tamizadas en Perú durante el 2012 (13).

Considerando que el VIH es el agente infeccioso con mayor impacto en salud pública, su escrutinio como potencial agente de contagio por vía transfusional es trascendental (11).

En relación al VIH, los estudios tanto de Colombia y Perú muestran valores de prevalencia similares $0,17 \%$ y $0,19 \%$ respectivamente a diferencia de Cuba 2,3\%

En relación al VHB, los estudios de México, Colombia muestran prevalencias similares $0,15 \%$ y $1,5 \%$ respectivamente a diferencia de los estudios peruanos que tienen prevalencias de $4,63 \%$ y $5,2 \%$.

En relación al $\mathrm{VHC}$, los estudios de México, Colombia y Perú muestran valores de prevalencia similares 0,57\%, $0,46 \%, 0,73 \%$ respectivamente a diferencia de Cuba que muestra una prevalencia de 2,3\%.

En relación a la infección por sífilis los estudios tanto de Colombia, Cuba y Perú muestran prevalencias similares $1,73 \%, 2,3 \%$ y $1,78 \%$ respectivamente.

La prevalencia encontrada para HTLV $(0,98 \%)$, porcentaje establecido por la OPS, para donantes peruanos (13).

En relación al HTLV, el estudio de Colombia muestra una prevalencia de $0,21 \%$ a diferencia de Perú que tiene una prevalencia de $1,21 \%$

\section{CONCLUSIONES}

Las cifras de prevalencia reportadas en los estudios son coherentes con los reportes de la OPS y coinciden entre ellos.

La prevalencia constante de estos marcadores se debe a la poca educación sanitaria al respecto de las enfermedades transmisibles en cuanto a su transmisibilidad, diagnóstico y tratamiento oportuno.

Reducir la prevalencia hallada en los donadores de sangre sigue siendo un reto importante de los Bancos de sangre, para mejorar la calidad de la transfusión, disminuir el costo económico y por tanto, asegurar la transfusión de sangre segura y oportuna a los pacientes. Además de captar donantes con un perfil que asegure la sangre segura, se debe tener en cuenta la disposición de los reactivos de tamizaje, continuo abastecimiento de los mismos y con un eficiente sistema de control de calidad. 


\section{REFERENCIAS BIBLIOGRÁFICAS}

1. Delgado MB. Transfusión sanguínea. Uso racional. Revista Colombiana de Anestesiología. Colombia 2012; 40(4): 247-248.

http://www.scielo.org.co/pdf/rca/v40n4/v40n4a01.pd $f$

2. Daza N, Sánchez M, Vanegas T, Ortega I. Prevalencia de infecciones en donantes de sangre en la Universidad Industrial de Santander versus parques de la ciudad de Bucaramanga, 2014. Revista de los estudiantes de medicina de la Universidad Industrial de Santander. Colombia 2016; 23(3): 55-60. http://www.scielo.org.co/pdf/muis/v29n3/0121-0319 -muis-29-03-00055.pdf

3. Rojo J. Enfermedades infecciosas transmitidas por transfusión. Panorama internacional y en México. Gaceta Médica de México. México 2014; 150(1): 78-83. http://www.anmm.org.mx/bgmm/2014/1/GMM_150_ 2014_1_078-083.pdf

4. Sánchez $P$, Sánchez MJ, Hernández S. Las enfermedades infecciosas y la transfusión de sangre. Revista Latinoamericana y Patología Clínica y Medicina de Laboratorio. Cuba 2012; 59(4): 186-193. http://www.medigraphic.com/pdfs/patol/pt-2012/pt1 24c.pdf

5. Concepción M, Concepción L, Marchena M, Estrada L. Frecuencia de marcadores serológicos de infecciones transmisibles por transfusión sanguínea en donantes voluntarios en un hospital de Trujillo, Perú. Revista del Cuerpo Médico del Hospital Nacional Almanzor Aguinaga Asenjo. Perú 2014; 7(3): 18-22.

http://www.imbiomed.com.mx/1/1/articulos.php?met hod $=$ showDetail\&id_articulo $=103389$ \&id_seccion $=4$ 615\&id_ejemplar=10046\&id_revista $=299$.

6. Loyola EO, Paredes JA. Digitalizando los datos en la gestión de donantes de sangre: ¿Lujo o necesidad?. Revista Médica Herediana. Perú 2014; 25(1): 53-54. http://www.scielo.org.pe/pdf/rmh/v25n1/v25n1cedit1 .pdf

7. J.Rojo-medina, JMBello-López. National prevalence of hepatitis C and B viruses in Mexican blood donors. Revista Médica del Hospital General de México. Mexico 2017; 80(1):37-44.
https://www.sciencedirect.com/science/article/pii/S 0185106316300749

8. Leidy Camargo De la Hoz, Claudia Consuegra, Astrid Coronado, et al. Perfil de donantes de un banco de sangre de la ciudad de Barranquilla- Colombia. Archivos Venezolanos de Farmacología y Terapéutica. 2018; 37(3).

http://www.revistaavft.com/images/revistas/2018/a vft_3_2018/9_perfil_donantes.pdf

9. Jaiberth Antonio Cardona, Jennifer Flórez Duque. Prevalencia de Virus de las Hepatitis B y C y Factores Asociados en un Banco de Sangre de Medellín. Archivos de medicina. Colombia 2018; 14(2):11.

10. María Antonia Ramos Ríos, Eraida Hernández Díaz, Osvaldo Miranda Gómez, et al. Incidencia de marcadores serológicos en donantes de sangre. Revista Cubana de Medicina Militar. Cuba 2014; 43(4):441-448

http://www.archivosdemedicina.com/medicina-de-f amilia/prevalencia-de-virus-de-las-hepatitis-b-y-c-y -factores-asociados-en-un-banco-de-sangre-de-me delliacuten-colombia-20152016.php?aid=22896

11. Jeél Moya, Edward Julcamanyan. Seroprevalencia de marcadores infecciosos causantes de pérdidas de hemodonaciones en el Servicio de Banco de Sangre del Hospital Nacional Docente Madre Niño San Bartolomé. Horiz Med. Perú 2014; 14(4): 6-14. http://www.scielo.org.pe/pdf/hm/v14n4/a02v14n4.p df

12. Morales J, Fuentes-Rivera J, Delgado-Silva C, Matta-Solís $H$. Marcadores de infección para hepatitis viral en donantes de sangre de un hospital nacional de Lima metropolitana. Rev Peru Med Exp Salud Pública. Perú 2017; 34(3):466-71. http://www.scielo.org.pe/scielo.php?script=sci_artte xt\&pid=S1726-46342017000300013

13. Supply of Blood for transfusion in the Caribean and Latin American Countries 2012-2013. Washington, DC. Organización Panamericana de la Salud, 2015. https://www.paho.org/hq/index.php?option=com_c ontent\&view $=$ article\&id =8918:2013-supply-blood-t ransfusion-caribbean-latin-american-countries-201 0-2011\&ltemid $=2163$ \&lang $=$ en

\section{PANACEA}

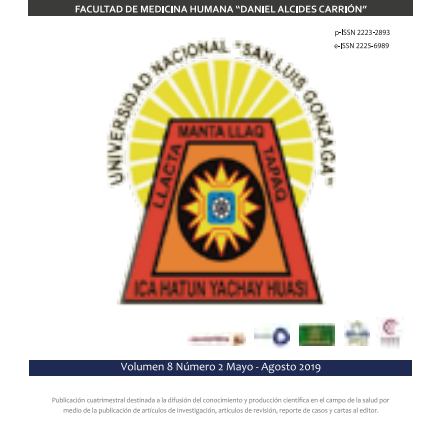

